\title{
Variation in the ability of fungi in the extrafloral nectar of Mallotus paniculatus to attract ants as plant defenders
}

\section{Sun PF, Chen PH, Lin WJ, Lin CC and Chou JY*}

\author{
Department of Biology, National Changhua University of Education, Changhua 500, Taiwan
}

Sun PF, Chen PH, Lin WJ, Lin CC, Chou JY 2018 - Variation in the ability of fungi in the extrafloral nectar of Mallotus paniculatus to attract ants as plant defenders. Mycosphere 9(2), 178188, Doi 10.5943/mycosphere/9/2/2

\begin{abstract}
Many plant-ant interactions are considered mutualisms. In Mallotus paniculatus (Euphorbiaceae) (also known as Turn-in-the-wind), the extrafloral nectaries (EFNs) on the base of the leaf laminas can produce sugar-rich secretions to attract ants as effective agents against herbivores or plant competitors. Growing evidence reveals that microorganisms are important "hidden players" in insect-plant interactions. Understanding which microorganisms act as such third-party species and how they operate is a major challenge in the study of mutualistic interactions. In this study, we showed that two dominant fungal species, the yeast Jaminaea angkorensis and the hyphal fungus Gibellulopsis nigrescens both from EFNs and the interiors of bodies of the ant Pheidole megacephala (Formicidae) on their own were sufficient for ant attraction. Our results also revealed that different fungal species on the host plant influenced ant behavior differently. These results can be applied in agriculture to increase ants or herbivore predators to protect plant hosts using fungal baits. Moreover, they indicate that fungal odors represent the critical signal to establish the plant-microbe-insect interactions. The traditional plantdefender concept must be updated to include the role of microorganisms.
\end{abstract}

Keywords - plant-ant interaction - extrafloral nectar - mutualism - olfaction - yeast

\section{Introduction}

Olfaction plays an important role in many behavioral contexts in insects. Insects receive odors emitted from individuals of the same species, such as sex pheromones for mate assessment (Johnson et al. 2017, Li et al. 2017), alarm pheromones for warning or alerting another of the same species of impending danger (Mizunami et al. 2010), and aggregation pheromones for attracting insects to favorable locations (Leonhardt et al. 2016). The insects also receive odor signals from other organisms such as enemies, potential hosts, preys, and food source (Gadenne et al. 2016). To successfully perform these behaviors, insects modulate their olfactory system according to their physiological state upon interaction with the environment. The changes in olfactory sensory pathways may even contribute to sympatric speciation (Scheidler et al. 2015). For example, Rhagoletis pomonella, apple maggot, is the pest of the apple tree. Studies have indicated that the shifts in neuronal pathways carrying information of behavioral relevance may represent a general phenomenon contributing to rapid shifts in habitat and mate choice, potentially catalyzing speciation (Linn et al. 2003). 
Plants also release pheromone cues that dictate insect behaviors. For example, the carnivorous plant Dionaea muscipula attracts insects to visit the deadly capture organ of flyflap by mimicking fruit and flower smells (Kreuzwieser et al. 2014). Maize (Zea mays) defends itself from pest feeding and egg deposition by emitting herbivore-induced plant volatiles. These volatiles attract parasitic wasps to attack moth eggs (Tamiru et al. 2017). Although other organisms can influence insect behavior by producing volatile organic compounds, only few studies have investigated the role of such compounds in interactions between symbiotic microorganism on the plants and insects (Aleklett et al. 2014). Symbiotic microbes on the plant host can help defend against herbivores by interfering with the chemical compounds produced by the host. The plant Arabidopsis thaliana produces volatiles in response to herbivory to defend itself against herbivorous insects. Pineda et al. showed that the volatile blend from rhizobacteria-treated aphidinfested Arabidopsis plants is less attractive to an aphid parasitoid than the volatile blend from aphid-infested plants without rhizobacteria (Pineda et al. 2013).

Ants are arguably the greatest success story in the history of terrestrial metazoa. They monopolize $15 \%-20 \%$ of the terrestrial animal biomass, particularly in tropical regions (Schultz 2000). Because of their widespread presence, they have different ways of interacting with other organisms. Many plants in the families Euphorbiaceae and Fabaceae have glands that secrete nectar and attract nearby ants to be their day-and-night patrol for mutualism. This is a plant-ant facultative mutualistic association (Pemberton 1998). In general, extrafloral nectar (EFN) is rich in sugars with small quantities of amino acids, especially essential amino acids. Because yeasts are common inhabitants of angiosperm floral nectar (FN) and EFN, they may act as causal agents of fermentation odors in plants and, therefore, as possible intermediate agents in plant signaling to pollinators (e.g., bees) or antiherbivory defense (e.g., ants) (Goodrich et al. 2006, Schaeffer et al. 2017). Nevertheless, studies on the potential role of yeasts in the origin of FN or EFN scents generally have not directly explored their presence or abundance in these two niches.

Mallotus paniculatus (Euphorbiaceae) (also known as Turn-in-the-wind) is a common tree at secondary forest edges and can be easily identified by its leaf morphology. It is distributed throughout India, Burma (Myanmar), Indo-China, southern China, Taiwan, and Thailand (Plants of the world online, http://powo.science.kew.org/; searched on 27. February 2018). The leaves have ovate laminas with pointed tips, white undersides, and long petioles (Fig. 1A). The lamina margins are simple to 3-lobed. The base of the leaf lamina has a pair of extrafloral glands and secretes a sugary fluid that attracts ants (Fig. 1B); this may be a mutualistic relationship, with the ant protecting the plant against herbivorous insects in return (So 2004). Thus, our aim was to provide critical empirical evidence in support of the hypothesis of fungus-mediated signaling in plant-ant interactions on the plant $M$. paniculatus. Herein, we show that fungi isolated from EFN on their own are sufficient for ant attraction and that ants behave differently to different fungus baits.

\section{Materials and methods}

\section{Study sites}

We conducted this study in Changhua County $\left(24^{\circ} 02^{\prime} 30.3^{\prime \prime} \mathrm{N}, 120^{\circ} 35^{\prime} 43.8^{\prime \prime} \mathrm{E}\right)$ in central Taiwan. The annual average temperature of Changhua is $22.4^{\circ} \mathrm{C}$, with July being the hottest month and January being the coolest. The annual average rainfall is $1723.4 \mathrm{~mm}$. The study site was located on a gentle slope in the Tri-Mountain National Scenic Area, an open shrubland dominated by $M$. paniculatus.

\section{Yeast collection}

We observed a greater nectar secretion rate in EFNs of young M. paniculatus individuals than in older plants, indicating that they are possibly more protected by ants. In addition, nectar secretion was higher at early morning (approximately 5 a.m. to 7 a.m.), a pattern that could be associated with an increase in herbivore pressure and water stress during the daylight hours. Thus, we collected the liquid secretion in EFN of three young $M$. paniculatus individuals by using a $0.2-$ 
$2-\mu \mathrm{L}$ pipette, which was immediately put in a sterilized 2-mL microcentrifuge tube. The samples were maintained at $4{ }^{\circ} \mathrm{C}$ until yeast isolation procedures. After the samples were transported to the laboratory, $100 \mu \mathrm{L}$ of sterilized water was added to the microcentrifuge tubes and vortexed for 5 min at 3,000 revolutions/min (rpm) using a vortex mixer (AL-VTX3000L, CAE technology Co., Ltd.), and the samples were spread uniformly by using sterile $4 \mathrm{~mm}$ glass beads on the YPD agar plates (1\% yeast extract, $2 \%$ peptone, $2 \%$ dextrose, and $2 \%$ agar) supplemented with approximately $2-3 \mathrm{~mL}$ of $100 \%$ lactic acid. The YPD plates were then incubated at $28^{\circ} \mathrm{C}$. Purified yeast strains were suspended in YPD medium supplemented with $15 \% \mathrm{v} / \mathrm{v}$ glycerol and maintained at $-80^{\circ} \mathrm{C}$.
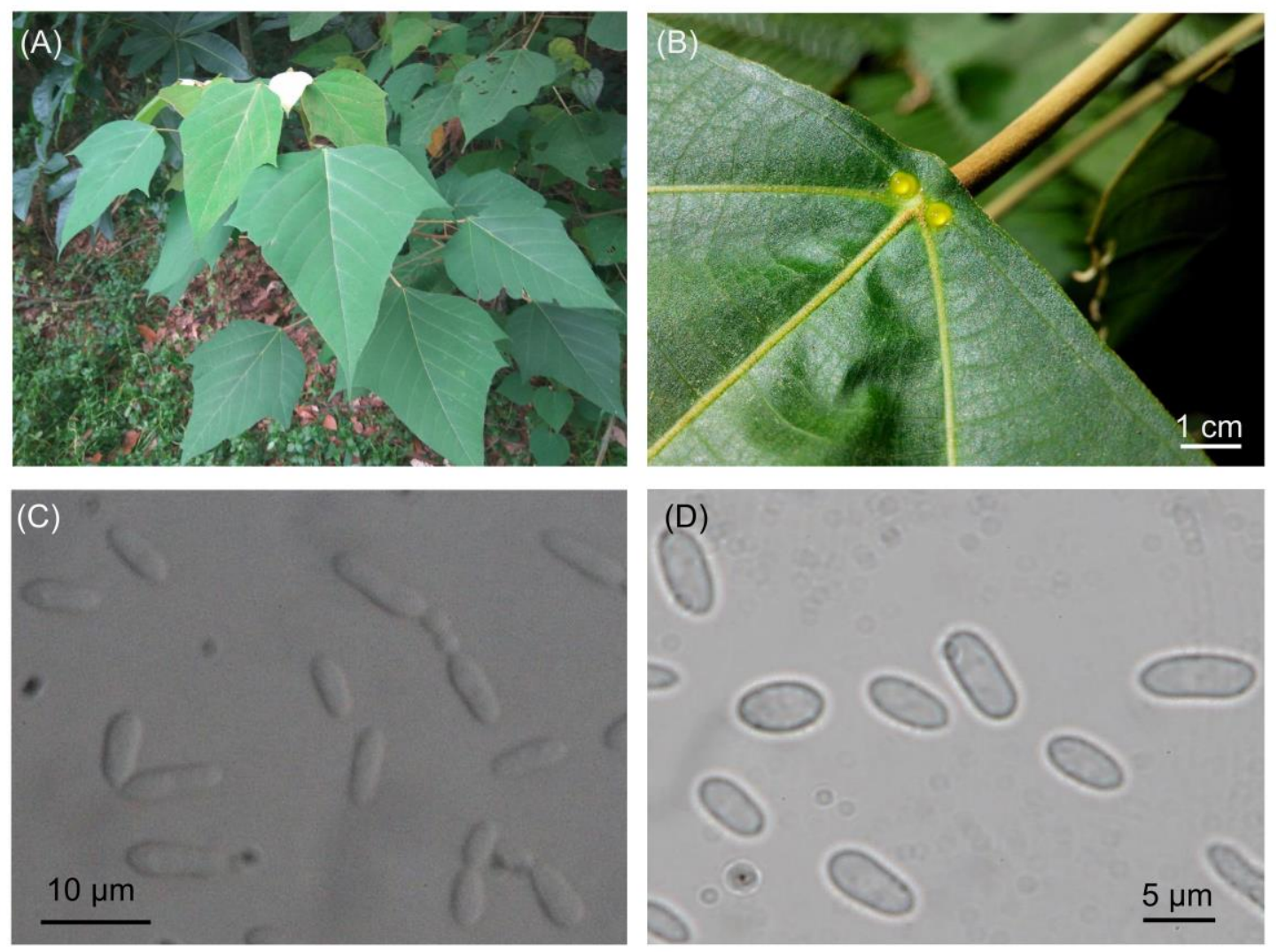

Figure 1 - (A) Characteristic leaves of the plant Mallotus paniculatus (also known as Turn-in-thewind). (B) Extrafloral nectaries of $M$. paniculatus at the base of the upper leaf lamina. (C) Microscopic morphology of the yeast Jaminaea angkorensis used as bait here. (D) Microscopic morphology of conidia of the hyphal fungus Gibellulopsis nigrescens used as bait here.

Three defender ants were also transported to the laboratory and then frozen at $-20^{\circ} \mathrm{C}$ to kill them. The corpses were transferred into sterilized microcentrifuge tubes $(2 \mathrm{~mL})$ with $70 \%$ ethanol to sterilize their body surface. They were then washed twice with $500 \mu \mathrm{L}$ of sterilized water, and the liquid was removed. Subsequently, $200 \mu \mathrm{L}$ of sterilized water was added, and the supernatant was spread on the YPD plate by gentle shaking to confirm the absence of any surviving yeast on the surface of the ants after sterilization. Finally, the ants were homogenized in $200 \mu \mathrm{L}$ of water, and the mixture was spread on the YPD plate to obtain the yeasts from the interiors of the ant bodies. The YPD plates were then incubated at $28^{\circ} \mathrm{C}$. Purified yeast strains were suspended in YPD medium supplemented with $15 \% \mathrm{v} / \mathrm{v}$ glycerol and maintained at $-80^{\circ} \mathrm{C}$.

\section{Yeast identification}

Yeast cultures $(1 \mathrm{~mL})$ were transferred to a $1.5-\mathrm{mL}$ microcentrifuge tube and centrifuged at 13,000-16,000 $\mathrm{g}$ for $1 \mathrm{~min}$. The supernatant was discarded, and the cell pellet was suspended in $200 \mu \mathrm{L}$ of lysis buffer (2\% Triton X-100, 1\% sodium dodecyl sulfate, $100 \mathrm{mM}$ sodium chloride [NaCl], $10 \mathrm{mM}$ Tris [pH 8.0], and $1 \mathrm{mM}$ ethylenediaminetetraacetic acid [EDTA]). Subsequently, 
$200 \mu \mathrm{L}$ of phenol:chloroform:isoamyl alcohol (25:24:1 [the isoamyl alcohol being optional]) and $0.3 \mathrm{~g}$ of acid-washed glass beads $(0.45-0.52 \mathrm{~mm})$ were added and gently mixed. The samples were vortexed for $5 \mathrm{~min}$ to disrupt cells and then centrifuged at 13,000-16,000 $\mathrm{g}$ for $5 \mathrm{~min}$. The aqueous layer of each sample was transferred to a clean tube, and $400 \mu \mathrm{L}$ of $95 \%$ ethanol and $16 \mu \mathrm{L}$ of $3 \mathrm{M}$ sodium acetate $(\mathrm{pH} 5.2)$ were added. The samples were mixed by inversion and centrifuged at $13,000-16,000 \mathrm{~g}$ for $5 \mathrm{~min}$. The pellets were washed with $300 \mu \mathrm{L}$ of $70 \%$ ethanol, and the samples were centrifuged at 13,000-16,000 $g$ for 2 min before the supernatant was discarded. Ethanol was aspirated for 30 min to dry the pellets. Finally, the genomic DNA from each sample was suspended in $100 \mu \mathrm{L}$ of Tris-EDTA buffer (pH 8.0).

Yeast identification was performed by sequencing the polymerase chain reaction (PCR) products of large subunit (LSU) rDNA, including the D1/D2 domain, 5.8S rDNA, and internal transcribed spacer (ITS) regions amplified by the universal primers ITS-1 (5'TCCGTAGGTGAACCTGCG-3') and NL-4 (5'-GGTCCGTGTTTCAAGACGG-3') (Kurtzman \& Robnett 1997). PCR proceeded as follows: initial denaturation at $95^{\circ} \mathrm{C}$ for $5 \mathrm{~min}$ followed by repeated denaturation at $95^{\circ} \mathrm{C}$ for $1 \mathrm{~min}$, annealing at $53^{\circ} \mathrm{C}$ for $30 \mathrm{~s}$, and elongation at $72^{\circ} \mathrm{C}$ for 1 min $40 \mathrm{~s}$ for 35 cycles; the final elongation lasted $5 \mathrm{~min}$. DNA sequencing of these samples was performed at Tri-I Biotech, Inc. A BLAST search of the nucleotide sequences was conducted through the National Center for Biotechnology Information homepage (http://www.ncbi.nlm.nih.gov). The voucher specimens of fungi used in this study are deposited in the Bioresource Collection and Research Center, Hsinchu City, Taiwan (http://www.bcrc.firdi.org.tw/). Any requests should be addressed to the corresponding author.

\section{Behavioral assays by yeast bait attraction}

To evaluate the variation in the ability of yeasts in EFN of $M$. paniculatus to attract ant defenders, we randomly selected $M$. paniculatus individuals. All selected plants were at least $10 \mathrm{~m}$ apart. Three randomly selected plants served as replicates in each experiment.

To prepare yeast cells for the attraction assay, actively growing yeast cells cultured in YPD medium at $28^{\circ} \mathrm{C}$ for approximately 12 hours were collected. The inocula were adjusted by YPD medium to an optical density of 0.8 at $660 \mathrm{~nm}$. We spotted $20 \mu \mathrm{L}$ of yeast culture or YPD medium only on the center between the midrib and leaf margin for the binary choice assay (Fig. 2). Furthermore, to investigate the difference between volatiles produced by young and old yeast cultures in terms of their attractiveness, actively growing yeast cells and 3-day-cultured (old, stationary stage) yeasts were used for comparison. The experiments were recorded using digital video recorder (SONY HDR-CX430V) to document the ant behavior until one of the baits was consumed completely. Once the baits were consumed completely, the ants dismissed and then returned to the nest. Thus, we can conclude that the baits were "consumed completely" by the ant behavior. The movie was divided into three equal parts (first, middle, and last) to analyze the differences between the responses of ants in different periods during the whole attraction assay. Two types of ant behaviors were analyzed. Attraction rate: We calculated the number of ants going into the counting area (the length between the midrib and leaf margin as the diameter) during the different periods (Fig. 2). We then divided the number of ants attracted in each period by the total number of ants attracted in the whole assay procedure to compare the attraction index between two baits. Stay rate: We calculated the difference between the number of ants attracted in each period and the number of ants left in each period and divided this difference by the number of ants attracted in each period.

\section{Data analysis}

Data are expressed as mean \pm standard deviation. The significance of differences between groups was determined using Student $t$ tests and analysis of variance. $P<0.05$ was considered statistically significant. 


\section{Results}

\section{Yeast and ant species used}

The total counts of yeast associated with the glands and ants can be determined by colony forming units (CFUs) on agar plates. In this study, we found two yeast species to be dominant in both EFN of M. paniculatus and the interiors of the ant bodies-Jaminaea angkorensis (accession number: MF765802) (Fig. 1C) and Gibellulopsis nigrescens (MF765803) (Fig. 1D). J. angkorensis is an anamorphic basidiomycetous yeast species originally isolated from decaying leaves in the world heritage site Angkor, Cambodia. Taxonomically, J. angkorensis is affiliated with Microstromatales (Exobasidiomycetes, Ustilaginomycotina, Basidiomycota) and represents a basal phylogenetic lineage of this fungal order (Sipiczki \& Kajdacsi 2009). G. nigrescens was originally described on potato tubers in England (Zare et al. 2007). The ant species in this study was Pheidole megacephala, judging by morphological characteristics.

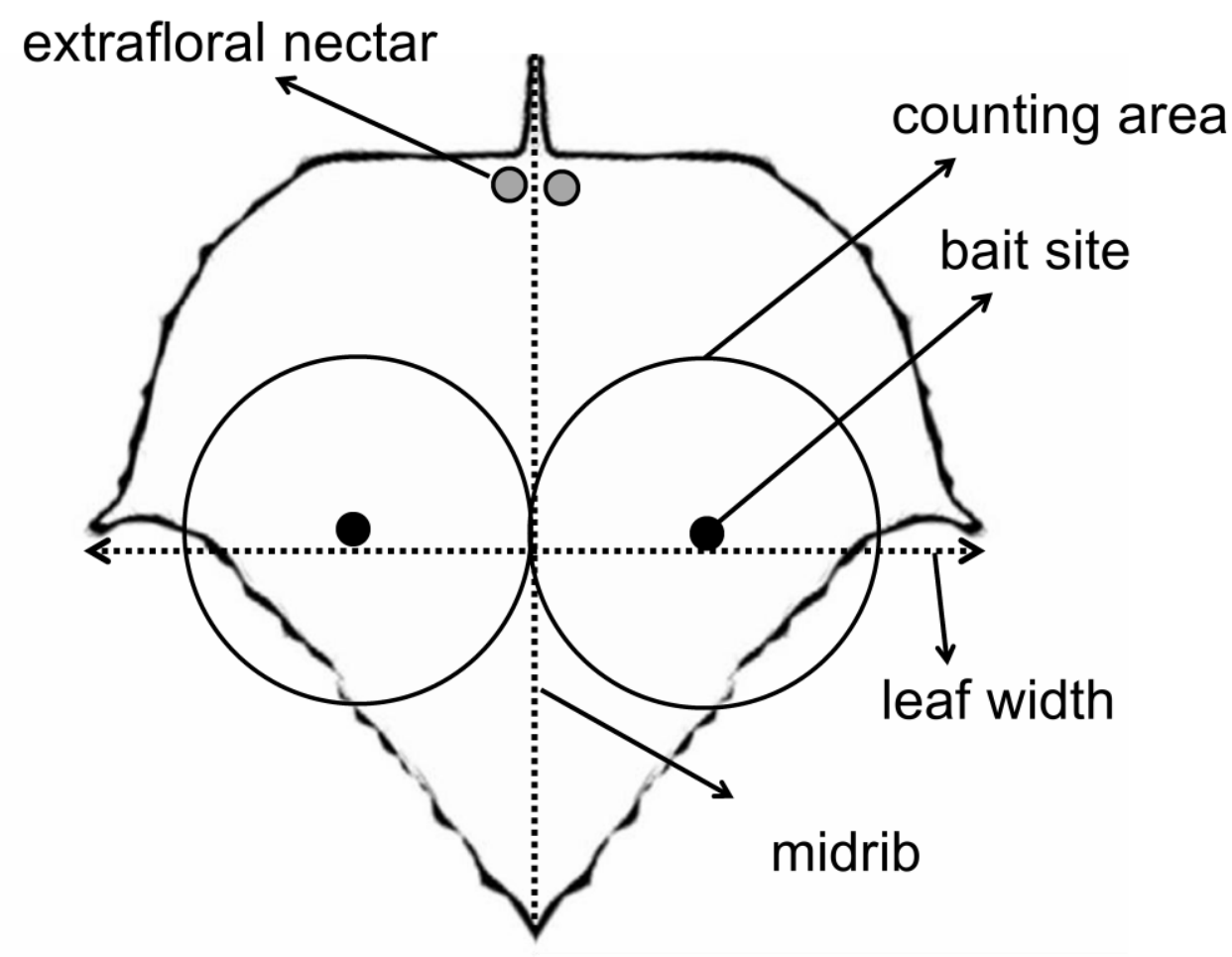

Figure 2 - Schematic of binary choice assay. The baits (yeast culture + YPD medium or pure YPD medium) were spotted on the center between the midrib and leaf margin for binary choice assay. Only ants going in the counting area (the length between the midrib and leaf margin as the diameter) were counted in this assay.

\section{Ant behavior}

\section{Attraction rate:}

In the binary choice assay between yeast + YPD medium and YPD medium alone, we found that both yeast species could significantly attract more ants than YPD medium. The average ant attraction rate for $J$. angkorensis was $56 \% \pm 2 \%$, which was higher than that for the control medium $(44 \% \pm 2 \%)$ (Fig. 3A); however, a statistically significant difference between the two was found only during the last period (Table 1). The ant attraction rate of G. nigrescens was $57 \% \pm 2 \%$ and that of YPD medium was $43 \% \pm 2 \%$ (Fig. 3B); however, a statistically significant difference was found only during the middle period (Table 1). Regarding the ability of attraction, G. nigrescens $(56 \% \pm 5 \%)$ attracted significantly more ants than J. angkorensis $(44 \% \pm 5 \%)$ (Fig. 3C), indicating 
that ants preferred G. nigrescens when both J. angkorensis and G. nigrescens yeasts were present. However, a statistically significant difference between $G$. nigrescens and J. angkorensis was found only during the middle period (Table 1 ).

We found that older yeast attracted significantly fewer ants than younger yeast. Comparing the 1-day- and 3-day-cultured yeast $J$. angkorensis, we found that the ant attraction rate of the younger yeast $(65 \% \pm 6 \%)$ was higher than that of the older yeast $(35 \% \pm 6 \%)$ (Fig. 3D). A statistically significant difference was found during the middle and last periods (Table 1). For $G$. nigrescens, the ant attraction rate of the 1-day-cultured yeast $(59 \% \pm 2 \%)$ was higher than that of the 3-day-cultured yeast $(41 \% \pm 2 \%)$ (Fig. 3E). A statistically significant difference was found only during the last period (Table 1). On comparing the attraction rate between the 3 -day-cultured yeasts of $J$. angkorensis $(49 \% \pm 7 \%)$ and $G$. nigrescens $(51 \% \pm 7 \%)$, we observed no significant difference (Fig. 3F); this indicates that ants did not exhibit bait preference for 3-day-cultured yeasts. Moreover, ants were less attracted to older yeasts.

\section{Stay rate:}

The average stay rate of $J$. angkorensis $(9 \% \pm 1 \%)$ was higher than that of the control medium $(5 \pm 1 \%)$ (Table 2). By contrast, the control medium had a higher average stay rate $(10 \pm$ $2 \%$ ) than $G$. nigrescens $(6 \pm 2 \%$ ) (Table 2$)$. In different periods of stay rate analysis, we noted a significant difference only in the late period of the assay between the control medium and yeast. No significant difference was found between J. angkorensis and G. nigrescens. In the experiment of different growth stages of yeasts, no difference in the stay rate was found between older and young yeasts.

\section{Direction preference assay}

In the previous experiment, ants exhibited different behaviors when they fed on different yeast baits. To determine whether ants had direction preference, we spotted YPD medium onto both spot sites on the leaf. Our results showed that ants did not have direction preference during the bait feeding experiment. Both left- and right-side baits attracted $50 \% \pm 2 \%$ of ants (Table 1), indicating that ants do not have direction preference when searching for food. In addition, we observed no difference in the stay rate between left- and right-side baits (Table 2).

\section{Discussion}

The study of plant-microbe-insect interactions has implications in fields of economic importance such as agriculture, biological invasion, and conservation (Biere \& Bennett 2013). Several important metabolic pathways are utilized by microorganisms to produce certain classes of end-product volatiles that have ecological relevance to insects (Davis et al. 2013). Among these microorganisms, yeasts have widespread associations with insect herbivores, and these interspecific interactions play a critical role in both yeast and insect evolution. Yeast cells produce various volatile metabolites that are key contributors to pleasing fruity and flowery aromas such as ethyl acetate (pear aroma), isoamyl acetate (banana aroma), and phenylethyl acetate (flowery aroma) (Pretorius et al. 1999, Nordström 1966, Saerens et al. 2008). Becher et al. (2012) revealed that yeast volatiles, rather than fruit volatiles, were principally responsible for the attraction of Drosophila melanogaster to food resources. Studies have also reported D. melanogaster to play a vital role in the ecosystem as an efficient vector for transfer of yeast species (Stamps et al. 2012, Reuter et al. 2007). Hunt \& Borden (1990) showed that two yeasts, Hansenula capsulata and Pichia pini, are responsible for the conversion of cis- and trans-verbenol to verbenone produced by female beetles (Dendroctonus ponderosae) and serves as an antiaggregation pheromone. Witzgall et al. (2012) found that the key pests of apple, codling moths (Cydia pomonella), are attracted to a fermenting yeast and lay more eggs on yeast-inoculated apples than on yeast-free apples. They also revealed that the yeast is an essential part of the larval diet and promotes larval survival by reducing the incidence of fungal infestations in the apple (Witzgall et al. 2012). Numerous studies have shown that yeasts can serve as a protein-rich food source for these insects and are beneficial to their 
development and immunocompetence (Anagnostou et al. 2010, Stamps et al. 2012). Insects can disperse yeasts through ingestion or carrying them externally (Reuter et al. 2007). Reuter et al. (2007) demonstrated that the outbreeding rate increased in Saccharomyces yeast in response to dispersal by fruit flies. Thus, a mutualistic interaction between these insect vectors and yeasts has been suggested (Witzgall et al. 2012).
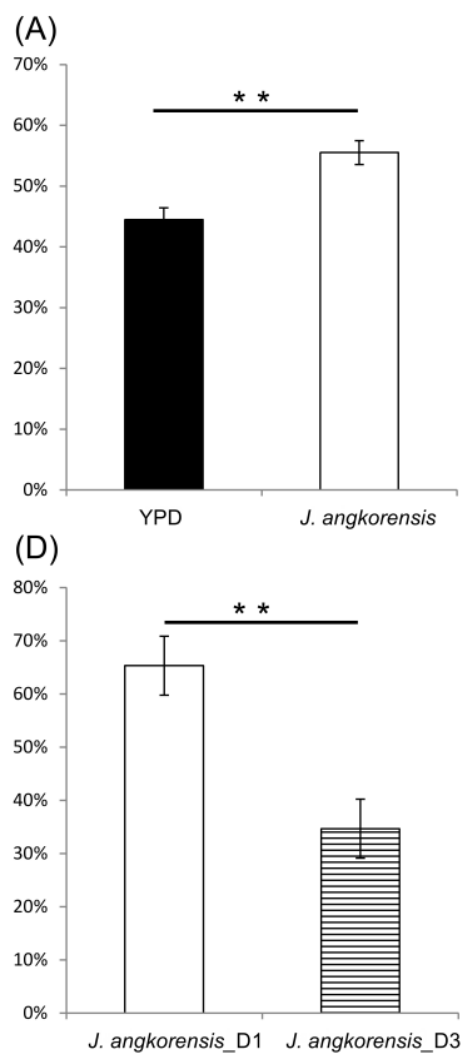

(B)

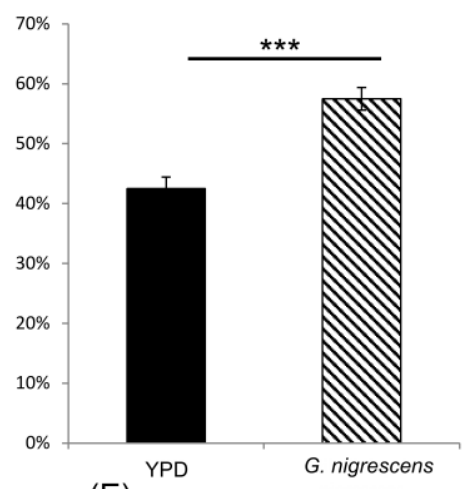

(E)

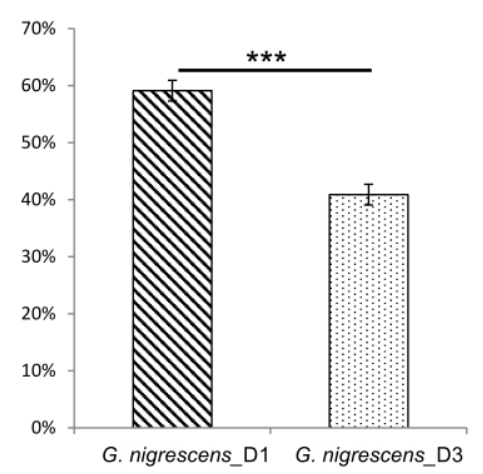

(C)

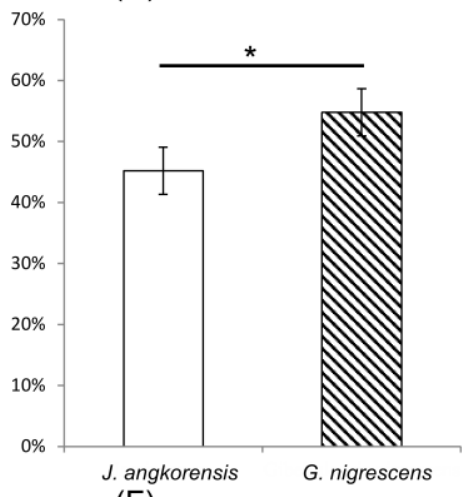

(F)

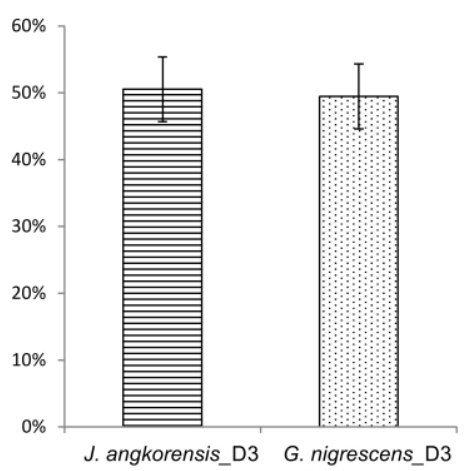

Figure 3 - Attraction rate of binary choice assay. (A) Jaminaea angkorensis compared with control medium. (B) Gibellulopsis nigrescens compared with control medium. (C) J. angkorensis compared with G. nigrescens. (D) 1-day-cultured J. angkorensis compared with 3-day-cultured $J$. angkorensis. (E) 1-day-cultured G. nigrescens compared with 3-day-cultured G. nigrescens. (F) 3day-cultured $J$. angkorensis compared with 3-day-cultured G. nigrescens. Data are expressed as mean \pm SD. $P<0.05$ was considered significant. * $P<0.05$; ** $P<0.01$; *** $P<0.001$

In the present study, we found that two fungal species were dominant both in EFN of $M$. paniculatus and the interiors of defender ant bodies; this indicates that the ants have close associations with these fungi. The ant feeding behavior presents the opportunity for acquiring fungi from EFN/or phyllosphere to the transmission of fungal population. For M. paniculatus, EFN defend plants indirectly by attracting patrolling ants that may reduce herbivory. The presence of ants on many EFN-bearing plants can reduce herbivory have been reported in many studies (reviewed in Heil 2015). Prof. Yamawo and his colleges have also indicated that the aggressiveness of ant species against herbivores on the EFN-bearing plant, M.japonicus and M. philippensis which are species close to M. paniculatus (Yamawo et al. 2012, 2014, 2017). The indirect anti-herbivore defense in symbiotic and non-symbiotic ant-plant associations in three Macaranga species have also been observed by Heil et al. (2001). Macaranga is a large genus of Old World tropical trees of the family Euphorbiaceae, the same as M. paniculatus. Although there is no direct evidence so far to show the defense specifically against herbivores by the ants on $M$. paniculatus, it is well accepted that the role of extrafloral nectaries of these plants is in attraction of ants and herbivore exclusion by ants (Rosumek et al. 2009, González-Teuber et al. 2012). Furthermore, in this study, we were unable to determine the importance of fungi to ant defenders. However, evidence suggests 
that yeasts transported by ants can change the sugar content of nectar (de Vega \& Herrera 2012, 2013). Thus, it may further change the pollination dynamics of ants or other pollinators. Mendes et al. demonstrated that yeasts growing in leaf-cutting ant nests may play the role of nutrient assimilation and removal of potentially toxic compounds that may be deleterious to the ants and their fungal cultivar (Mendes et al. 2012). This gives rise to intricate plant-microbe-insect interactions.

Table 1 Attraction rate of binary choice assay. D1 and D3 indicate 1-day-cultured (actively growing yeast cells) and 3-day-cultured (old, stationary stage) yeasts. Data are expressed as mean \pm SD. $P<0.05$ was considered significant. * $P<0.05$; ** $P<0.01$; *** $P<0.001$.

\begin{tabular}{lllll}
\hline Baits type & First $( \pm$ SD) & Middle $( \pm$ SD) & Last $( \pm$ SD) & Total \\
\hline YPD & $3 \%( \pm 2 \%)$ & $20 \%( \pm 4 \%)$ & $22 \%( \pm 4 \%)$ & $44 \%( \pm 2 \%)$ \\
J. angkorensis & $4 \%( \pm 3 \%)$ & $23 \%( \pm 1 \%)$ & $30 \%( \pm 5 \%)^{*}$ & $56 \%( \pm 2 \%)^{* *}$ \\
\hline YPD & $4 \%( \pm 4 \%)$ & $15 \%( \pm 1 \%)$ & $23 \%( \pm 4 \%)$ & $43 \%( \pm 2 \%)$ \\
G. nigrescens & $5 \%( \pm 5 \%)$ & $24 \%( \pm 3 \%)^{*}$ & $29 \%( \pm 5 \%)$ & $57 \%( \pm 2 \%)^{* * *}$ \\
\hline J. angkorensis & $7 \%( \pm 1 \%)$ & $16 \%( \pm 4 \%)$ & $20 \%( \pm 4 \%)$ & $44 \%( \pm 5 \%)$ \\
G. nigrescens & $9 \%( \pm 5 \%)$ & $24 \%( \pm 1 \%)^{*}$ & $25 \%( \pm 4 \%)$ & $56 \%( \pm 5 \%)^{*}$ \\
\hline J. angkorensis D1 & $10 \%( \pm 4 \%)$ & $28 \%( \pm 7 \%)^{*}$ & $28 \%( \pm 3 \%)^{* *}$ & $65 \%( \pm 6 \%)^{* *}$ \\
J. angkorensis D3 & $4 \%( \pm 3 \%)$ & $14 \%( \pm 0 \%)$ & $16 \%( \pm 3 \%)$ & $35 \%( \pm 6 \%)$ \\
\hline G. nigrescens D1 & $14 \%( \pm 10 \%)$ & $21 \%( \pm 8 \%)$ & $24 \%( \pm 5 \%)^{*}$ & $59 \%( \pm 2 \%)^{* * *}$ \\
G. nigrescens D3 & $11 \%( \pm 10 \%)$ & $16 \%( \pm 4 \%)$ & $15 \%( \pm 4 \%)$ & $41 \%( \pm 2 \%)$ \\
\hline J. angkorensis D3 & $8 \%( \pm 7 \%)$ & $19 \%( \pm 4 \%)$ & $26 \%( \pm 6 \%)$ & $49 \%( \pm 7 \%)$ \\
G. nigrescens D3 & $4 \%( \pm 4 \%)$ & $16 \%( \pm 4 \%)$ & $26 \%( \pm 5 \%)$ & $51 \%( \pm 7 \%)$ \\
\hline YPD Left & $9 \%( \pm 3 \%)$ & $18 \%( \pm 2 \%)$ & $24 \%( \pm 3 \%)$ & $50 \%( \pm 2 \%)$ \\
YPD Right & $8 \%( \pm 2 \%)$ & $16 \%( \pm 1 \%)$ & $25 \%( \pm 3 \%)$ & $50 \%( \pm 2 \%)$ \\
\hline
\end{tabular}

Table 2 Stay rate of binary choice assay. Data are expressed as mean \pm SD. $P<0.05$ was considered significant. $* P<0.05$; ** $P<0.01$.

\begin{tabular}{lllll}
\hline Baits type & First $( \pm$ SD) & Middle $( \pm$ SD) & Last $( \pm$ SD) & Total \\
\hline YPD & $46 \%( \pm 31 \%)$ & $16 \%( \pm 3 \%)$ & $10 \%( \pm 1 \%)$ & $5 \%( \pm 1 \%)$ \\
J. angkorensis & $53 \%( \pm 18 \%)$ & $17 \%( \pm 6 \%)$ & $17 \%( \pm 2 \%)^{*}$ & $9 \%( \pm 1 \%)^{* *}$ \\
\hline YPD & $46 \%( \pm 22 \%)$ & $23 \%( \pm 7 \%)$ & $18 \%( \pm 0 \%)^{*}$ & $10 \%( \pm 2 \%)^{*}$ \\
G. nigrescens & $47 \%( \pm 30 \%)$ & $21 \%( \pm 5 \%)$ & $13 \%( \pm 2 \%)$ & $6 \%( \pm 2 \%)$ \\
\hline J. angkorensis & $18 \%( \pm 6 \%)$ & $15 \%( \pm 7 \%)$ & $9 \%( \pm 2 \%)$ & $4 \%( \pm 1 \%)$ \\
G. nigrescens & $15 \%( \pm 7 \%)$ & $11 \%( \pm 7 \%)$ & $6 \%( \pm 3 \%)$ & $3 \%( \pm 1 \%)$ \\
\hline J. angkorensis D1 & $39 \%( \pm 8 \%)$ & $17 \%( \pm 10 \%)$ & $16 \%( \pm 9 \%)$ & $5 \%( \pm 3 \%)$ \\
J. angkorensis D3 & $30 \%( \pm 11 \%)$ & $14 \%( \pm 10 \%)$ & $17 \%( \pm 12 \%)$ & $5 \%( \pm 3 \%)$ \\
\hline G. nigrescens D1 & $22 \%( \pm 5 \%)$ & $21 \%( \pm 21 \%)$ & $18 \%( \pm 14 \%)$ & $7 \%( \pm 4 \%)$ \\
G. nigrescens D3 & $15 \%( \pm 9 \%)$ & $16 \%( \pm 11 \%)$ & $13 \%( \pm 13 \%)$ & $8 \%( \pm 6 \%)$ \\
\hline J. angkorensis D3 & $32 \%( \pm 30 \%)$ & $18 \%( \pm 6 \%)$ & $9 \%( \pm 1 \%)$ & $6 \%( \pm 4 \%)$ \\
G. nigrescens D3 & $16 \%( \pm 4 \%)$ & $12 \%( \pm 3 \%)$ & $8 \%( \pm 3 \%)$ & $4 \%( \pm 3 \%)$ \\
\hline YPD Left & $14 \%( \pm 3 \%)$ & $10 \%( \pm 3 \%)$ & $9 \%( \pm 4 \%)$ & $5 \%( \pm 2 \%)$ \\
YPD Right & $25 \%( \pm 20 \%)$ & $12 \%( \pm 9 \%)$ & $11 \%( \pm 5 \%)$ & $4 \%( \pm 1 \%)$ \\
\hline
\end{tabular}

Niche construction refers to the process by which an organism alters its own (or another species') environment and this modification affects it evolutionary trajectory. This intricate backand-forth process creates a feedback relationship between natural selection and niche construction. Thus, some biologists have argued that niche construction may be a general mechanism driving the evolution of mutualisms. Buser et al. (2014) revealed that the fruit fly Drosophila simulans was more attracted to $S$. cerevisiae yeast strains that were naturally associated with $D$. simulans when compared with other strains of $S$. cerevisiae. More attractive yeasts are dispersed more frequently, and the flies associated with more attractive yeasts have higher fecundity (Buser et al. 2014). 
Similarly, yeasts isolated from fruit-associated niches were more attractive than those from nonfruit-associated niches (Palanca et al. 2013). Because the fungal species used in the present study were isolated from $\mathrm{EFN}$ and interiors of ants, our findings suggest a general positive preference/attractiveness toward naturally associated fungi by ant defenders. However, only two fungal species isolated were used, and how differences in host ecology affect the ant-yeast relationship remains unknown. Determining whether fungi isolated from ant-associated environments are more attractive than those from other environments is appealing.

\section{Acknowledgments}

We thank the members of the Chou Lab for their contribution to the manuscript. This work was supported by grants from the Ministry of Science and Technology (MOST 104-2311-B-018001 and MOST 105-2311-B-018-001-MY3 to J.-Y. Chou). This manuscript was edited by Wallace Academic Editing.

\section{References}

Aleklett K, Hart M, Shade A. 2014 - The microbial ecology of flowers: an emerging frontier in phyllosphere research. Botany 92, 253-266.

Anagnostou C, LeGrand EA, Rohlfs M. 2010 - Friendly food for fitter flies?-Influence of dietary microbial species on food choice and parasitoid resistance in Drosophila. Oikos 119, 533-541.

Becher PG, Flick G, Rozpędowska E, Schmidt A et al. 2012 - Yeast, not fruit volatiles mediate Drosophila melanogaster attraction, oviposition and development. Functional Ecology 26, 822-828.

Biere A, Bennett AE. 2013 - Three-way interactions between plants, microbes and insects. Functional Ecology 27, 567-573.

Buser CC, Newcomb RD, Gaskett AC, Goddard MR. 2014 - Niche construction initiates the evolution of mutualistic interactions. Ecology Letters 17, 1257-1264.

Davis TS, Crippen TL, Hofstetter RW, Tomberlin JK. 2013 - Microbial volatile emissions as insect semiochemicals. Journal of Chemical Ecology 39, 840-859.

de Vega C, Herrera CM. 2012 - Relationships among nectar-dwelling yeasts, flowers and ants: patterns and incidence on nectar traits. Oikos 121, 1878-1888.

de Vega C, Herrera CM. 2013 - Microorganisms transported by ants induce changes in floral nectar composition of an ant-pollinated plant. American Journal of Botany 100, 792-800.

Gadenne C, Barrozo RB, Anton S. 2016 - Plasticity in insect olfaction: To smell or not to smell? Annual Review of Entomology 61, 317-333.

Goodrich KR, Zjhra ML, Ley CA, Raguso RA. 2006 - When flowers smell fermented: the chemistry and ontogeny of yeasty floral scent in pawpaw (Asimina triloba: Annonaceae). International Journal of Plant Sciences 167, 33-46.

González-Teuber M, Bueno JCS, Heil M, Boland W. 2012 - Increased host investment in extrafloral nectar (EFN) improves the efficiency of a mutualistic defensive service. PLoS One 7, e46598.

Heil M, Fiala B, Maschwitz U, Linsenmair KE. 2001 - On benefits of indirect defence: short-and long-term studies of antiherbivore protection via mutualistic ants. Oecologia 126, 395-403.

Heil M. 2015 - Extrafloral nectar at the plant-insect interface: a spotlight on chemical ecology, phenotypic plasticity, and food webs. Annual Review of Entomology 60, 213-232.

Hunt DW, Borden JH. 1990 - Conversion of verbenols to verbenone by yeasts isolated from Dendroctonus ponderosae (Coleoptera: Scolytidae). Journal of Chemical Ecology 16, 13851397.

Johnson TL, Symonds MR, Elgar MA. 2017 - Sexual selection on receptor organ traits: younger females attract males with longer antennae. The Science of Nature 5, 1-6.

Kreuzwieser J, Scheerer U, Kruse J, Burzlaff T et al. 2014 - The Venus flytrap attracts insects by the release of volatile organic compounds. Journal of Experimental Botany 65, 755-766. 
Kurtzman C, Robnett C. 1997 - Identification of clinically important ascomycetous yeasts based on nucleotide divergence in the 5'end of the large-subunit (26S) ribosomal DNA gene. Journal of Clinical Microbiology 35, 1216-1223.

Leonhardt SD, Menzel F, Nehring V, Schmitt T. 2016 - Ecology and Evolution of Communication in Social Insects. Cell 164, 1277-1287.

Li C, Wang H, Chen X, Yao J et al. 2017 - Role of visual and olfactory cues in sex recognition in butterfly Cethosia cyane cyane. Scientific Reports 7, 5033.

Linn C, Feder JL, Nojima S, Dambroski HR et al. 2003 - Fruit odor discrimination and sympatric host race formation in Rhagoletis. Proceedings of the National Academy of Sciences 100, 11490-11493.

Mendes TD, Rodrigues A, Dayo-Owoyemi I, Marson FA, Pagnocca FC. 2012 - Generation of Nutrients and Detoxification: Possible Roles of Yeasts in Leaf-Cutting Ant Nests. Insects 3, 228-245.

Mizunami M, Yamagata N, Nishino H. 2010 - Alarm pheromone processing in the ant brain: an evolutionary perspective. Frontiers in Behavioral Neuroscience 4, 28.

Nordström K. 1966 - Formation of Esters from Acids by Brewer's Yeast: Formation from Unsaturated Acids. Nature 210, 99-100.

Palanca L, Gaskett AC, Gunther CS, Newcomb RD, Goddard MR. 2013 - Quantifying variation in the ability of yeasts to attract Drosophila melanogaster. PLoS One 8, e75332.

Pemberton RW. 1998 - The occurrence and abundance of plants with extrafloral nectaries, the basis for antiherbivore defensive mutualisms, along a latitudinal gradient in east Asia. Journal of Biogeography 25, 661-668.

Pineda A, Soler R, Weldegergis BT, Shimwela MM et al. 2013 - Non-pathogenic rhizobacteria interfere with the attraction of parasitoids to aphid-induced plant volatiles via jasmonic acid signalling. Plant, Cell \& Environment 36, 393-404.

Pretorius I, Van der Westhuizen T, Augustyn O. 1999 - Yeast biodiversity in vineyards and wineries and its importance to the South African wine industry. A review. South African Journal of Enology and Viticulture 20:61-70

Reuter M, Bell G, Greig D. 2007 - Increased outbreeding in yeast in response to dispersal by an insect vector. Current Biology 17, R81-R83.

Rosumek FB, Silveira FA, de S Neves F, de U Barbosa NP et al. 2009 - Ants on plants: a metaanalysis of the role of ants as plant biotic defenses. Oecologia 160, 537-549.

Saerens S, Delvaux F, Verstrepen K, Van Dijck P et al. 2008 - Parameters affecting ethyl ester production by Saccharomyces cerevisiae during fermentation. Applied and Environmental Microbiology 74, 454-461.

Schaeffer RN, Mei YZ, Andicoechea J, Manson JS, Irwin RE. 2017 - Consequences of a nectar yeast for pollinator preference and performance. Fungal Biology 31, 613-621.

Scheidler NH, Liu C, Hamby KA, Zalom FG, Syed Z. 2015 - Volatile codes: correlation of olfactory signals and reception in Drosophila-yeast chemical communication. Scientific Reports 5, 14059

Schultz TR. 2000 - In search of ant ancestors. Proceedings of the National Academy of Sciences 97, $14028-14029$.

Sipiczki M, Kajdacsi E. 2009 - Jaminaea angkorensis gen. nov., sp. nov., a novel anamorphic fungus containing an S943 nuclear small-subunit rRNA group IB intron represents a basal branch of Microstromatales. International Journal of Systematic and Evolutionary Microbiology 59, 914-920.

So ML. 2004 - The occurrence of extrafloral nectaries in Hong Kong plants. Botanical Bulletin of Academia Sinica, 237-245.

Stamps JA, Yang LH, Morales VM, Boundy-Mills KL. 2012 - Drosophila regulate yeast density and increase yeast community similarity in a natural substrate. PLoS One 7, e42238.

Tamiru A, Bruce TJ, Richter A, Woodcock CM et al. 2017 - A maize landrace that emits defense volatiles in response to herbivore eggs possesses a strongly inducible terpene synthase gene. 
Ecology and Evolution 7, 2835-2845.

Witzgall P, Proffit M, Rozpedowska E, Becher PG et al. 2012 - "This is not an apple"-yeast mutualism in codling moth. Journal of Chemical Ecology 38, 949-957.

Yamawo A, Katayama N, Suzuki N, Hada Y. 2012 - Plasticity in the expression of direct and indirect defence traits of young plants of Mallotus japonicus in relation to soil nutritional conditions. Plant Ecology 213, 127-132.

Yamawo A, Tagawa J, Suzuki N. 2014 - Two Mallotus species of different life histories adopt different defense strategies in relation to leaf age. Plant species biology 29, 152-158.

Yamawo A, Hada Y, Tagawa J. 2017 - Aggressiveness of ants attracted to the extrafloral nectary-bearing plant, Mallotus japonicus, and temporal fluctuations in their abundance. Entomological Science 20, 150-155.

Zare R, Gams W, Starink-Willemse M, Summerbell R. 2007 - Gibellulopsis, a suitable genus for Verticillium nigrescens, and Musicillium, a new genus for $V$. theobromae. Nova Hedwigia 85, 463-489. 